\title{
Biochemical Systems Theory and Metabolic Control Theory: 1. Fundamental Similarities and Differences
}

\author{
MICHAEL A SAVAGF.AU, FBFR HARD O. VOIT, ${ }^{*}$ aND DOUGL.AS H. IRVINE \\ Department of Microbiology and Immunologt, The University of Michigan. \\ Ann Arbor, Michigan 48109
}

Receiced 11 August 1986; revised 18 June 1987

\begin{abstract}
Biochemical Systems Theory (BST) was developed in the late 1960s to explicate the integrated behavior of intact biochemical systems-specific dynamic behavior as well as general principles of design-in relation to the properties of their underlying molecular elements. This approach was used successfully in a number of biochemical and other biological applications throughout the 1970s and 1980s. A related approach. Metabolic Control Theory (MCT), was proposed in the mid 1970s. Its developments gencrally have followed without reference the analogous developments in BST, and its proponents have treated the two approaches as if they were unrelated. Detailed comparison of the fundamental structures of BST and MCT shows that. although there are some superficial differences, both in fact are based upon the same underlying formalism. Molecular descriptions in MCT comprise a special case of those in BST. Systemic descriptions differ with respect to the level of aggregation assumed. The aggregation at the level of net increase or net decrease of each system constituent found in BST is shown to produce the more revealing and useful theory. and results presented elsewhere [41] suggest that this level of aggregation also provides a more accurate description of the system. At this fundamental level, MCT represents a special case of BST, for the content and range of validity of BST are more inclusive than those of MCT.
\end{abstract}

\section{INTRODUCTION}

Several papers concerning the quantification of interactions within intact biochemical systems have appeared in the recent biochemical literature (e.g., see $[42,46,6])$. This work stemmed from two papers $[12,10]$ whose principal contribution was the demonstration of two relationships among local and global parameters of a biochemical system assumed to be in steady state: the

\footnotetext{
*Current address: Department of Biometry, Medical University of South Carolina. Charleston, SC 29425.
} 
summation relationships and the connectivity relationships. These relationships also have been called control theorems, and this approach to understanding intact biochemical systems has been named control theory [42], metabolic control theory [46], metabolic control [6], or control analysis [13]. ${ }^{1}$ This approach will be referred to here as MCT.

Nowhere in this body of work is there appropriate reference to an earlier theory [22-27] or its subsequent development (e.g., see [29-31, 33]) that deals with the same subject, namely, the behavior of intact biochemical systems. This earlier approach, called Biochemical Systems Theory, will be referred to here as BST. The principal contributions of BST were (1) a

\footnotetext{
${ }^{1}$ These are unfortunate choices for terms because a large established discipline called Control Theory, with numcrous journals, text books, conferences, training programs, etc., already exists. The opportunity for confusion is great because the major goals of this discipline, although representing a subset of goals that can be identified with the understanding of spccific control mechanisms in hinchemistry, are not the same as the more general goal of understanding all interactions within a biochemical system [1 $\mathrm{Ch} .1]$. The more general goal is associated with another large and well-established discipline called Systems Theory. Since it is the stated intention of workers in this field (e.g., Savageau [23, 27] and Kacser and Burns [12]) to develop a general theory that deals with all interactions and not just those associated with specific control mechanisms, identification with the general discipline of Systems Theory is more appropriate. Such an identification, aside from avoiding semantic confusion, also would promote the flow of useful techniques, concepts, etc., already established in the more general discipline into the fledgling discipline of Biochemical Systems Theory.

It is the ambiguity inherent in the use of the word "control," which has multiple meanings, that continues to plague effective communication in modern biology. For example, the proponents of MCT, in their use of the term "control" and their criticism of others" use of terms like "control enzyme," fail to make fundamental distinctions that go back to Aristotle: between material causes (components, mechanisms) and efficient causes (source of movements, initiating events) on the one hand, and between direct causes (primary effectors) and indirect causes (secondary effectors) on the other. The clear focus of modern biology is upon direct material causality. Most of what we know today about biochemical control has resulted from the identification of the underlying molecular mechanisms of control that are realized through the evolution of key enzymes, DNA sequences, etc. Any sound attempt to understand complex integrated systems will take this knowledge into account. It is counterproductive to "deny forcefully the usual textbook statement that such allosteric inhibition alone is the controlling element in a pathway" and to berate investigators who "persist in calling [thesel 'regulatory enzyme[s]" [13]. The ambiguity created by Kacser and Porteous insisting on the use of the term "control" (in the sense of indirect cause) over against the term "control" (in the sense of direct cause or mechanism) is most evident in their descriptions of fecdback inhibition; they conclude that the more effective the "control" enzyme (direct cause, mechanism) the less "control" (indirect cause) it exerts [13]. What these authors mean by "control" is more commonly referred to as "parameter sensitivity" in the existing literature on control theory and systems analysis (c.g., see [4.29 Ch. 9]).
} 
general and straightforward method of representing biochemical systems and (2) the derivation of an explicit steady-state solution in symbolic form relating each concentration variable and flux within an arbitrary system to external (or environmentally determined) concentration variables and parameters associated with the individual enzymes and processes of the system [23]. BST provided a direct answer to the question of how any concentration or flux is dependent upon the independent variables and parameters of the system. For convenience in relating molecular and systemic properties, these general answers also were expressed in terms of well-known global or systemic concepts: gain (or amplification) factors [25] and sensitivity coefficients $[25,26]$. BST was reviewed in 1972 and presented along with several applications to general classes of biochemical systems [27].

BST was largely complete for the steady-state domain and was published before the first papers on MCT appeared. It is clear from reading the original contributions to MCT that the same problem was addressed and that many of the same types of parameters and factors were defined. Subsequent developments in MCT have continued to follow, without reference, the analogous developments in BST [39]. This fact is difficult to understand, for the principal investigators of MCT were familiar with BST.

It is critical to eliminate any impediment to the full understanding of these theories and their ability to reveal the behavior of complex biochemical systems. We shall focus upon two issues that appear to be central to such clarification. First, the development of BST was presented in the context of a general mathematical formalism, while the development of MCT was presented in an ad hoc descriptive manner. This reason for the relatedness of the approaches being obscured should become less important with time because those interested in the behavior of integrated biochemical systems have begun to realize the importance of systematic mathematical tools for this task and because recent developments of MCT along these lines (e.g., see $[46,6])$ have brought it closer to BST. Second, and perhaps more important, the major achievements of MCT, namely the summation and connectivity relationships, are not apparent in BST. These relationships can be demonstrated readily within BST [29 Ch. 9]; however, they play no obvious role. Thus, what is most visible and central in one approach appears to be invisible and peripheral in the other. Perhaps this difference has caused subsequent workers to assume mistakenly that these two approaches lead to unrelated theories of biochemical systems. This is a serious issue that remains an obstacle to the recognition of their true similarities and differences and to a deeper understanding of complex biochemical systems.

In this first of two papers offered to rectify these misunderstandings we shall address the fundamental characteristics of BST and MCT. It will be demonstrated that the same mathematical structure, the Power-Law Formalism, underlies both BST and MCT, but that they differ with respect to 
implementation. First the Power-Law Formalism is described. Next it is shown that different approaches to the aggregation of fluxes and the aggregation of concentrations are characteristic of BST and MCT. The approach used in BST is responsible for its systematic character, and it leads naturally to an existence theorem for steady-state solutions and to a direct symbolic method of solution for the general steady-state behavior of biochemical systems. In each section we shall present first the development in BST, then, where applicable, the corresponding results in MCT, and finally a brief comparison.

In the second paper [34] we shall present the derivation of summation and connectivity relationships within BST and show how their role differs from that within MCT. In a third paper [41], the relative accuracies of BST and MCT are compared, and BST is shown to provide a more accurate representation of biochemical systems.

\section{POWER-LAW FORMALISM}

In many cases individual enzyme-catalyzed reactions in quasi-steady state are assumed to be appropriate elemental components of biochemical systems. Nevertheless, there are well-known systems for which this assumption is invalid. For example, a number of systems involve association and dissociation of enzymes (e.g., see [47, $29 \mathrm{Ch} .12,17,35,16,8,43-45]$ ) or consist of multifunctional enzymes that catalyze several reactions (c.g., see $[20,5,9,14,36])$. In the first example, the traditional assumptions of the Michaelis-Menten Formalism do not apply $[32,41]$ and there may be no simple mathematically expressible rate law for the enzyme. In some instances, the overall reaction may be nonlinearly dependent on gene dose, enzyme concentration, or molecular activity; e.g., the reaction rate of an enzyme with dimeric associations may exhibit a quadratic dependence on gene dose. The rate law for one enzyme may be a complex function of the concentration of another enzyme or of its activity. Here simple chemical reactions, or a subset of the chemical kinetic pathways that constitute the enzymatic mechanism, might be a more natural elemental component of the overall system. Constraints among these elements (see Section 3.2) generally must be considered. In the second example, several reactions may function as a unified enzyme-kinetic mechanism or channel $[5,9,43,37]$, and the entire group of reactions might constitute a natural elemental component of the overall system. In still other examples, enzymes that catalyze nonconsecutive reactions [20,29 Ch. 12,36] or that have a subunit in common but are located in different pathways [14] are constrained to operate in a similar fashion. These considerations illustrate how the kinetic behavior of enzymes in situ may be considerably more complex than is indicated in most textbook accounts. 
Even if one ignores these difficulties and considers only the usual assumptions, it has long been recognized that detailed kinetic description of biochemical processes leads to nonlinear functions that are too complicated to be of practical use or to form the basis of a general formalism for complex biochemical systems with many enzymes. The intractability of the differential equations describing systems that involve such nonlinearities led to the search for a general formalism that would retain the essential nonlinear features and yet be amenable to mathematical analysis (for detailed discussion, see $[23,27])$.

\subsection{DERIVATION}

In searching for an appropriate general formalism to characterize complex biochemical systems, one cannot avoid being influenced by the paradigm of linear systems. The linear formalism is attractive because it is a general symbolic formalism, because it offers powerful mathematical methods for analysis, and because there are cases of its successful application in biochemistry. However, it has distinct disadvantages in that it cannot adequately represent most biochemical systems, which are nonlinear. It is known to be incapable of representing important behavior that arises from the nonlinearities - thresholds, saturation, synergism, memory, limit cycles, chaos, etc. Its use as an approximation for these systems is severely limited because its valid region is restricted to a narrow range of values for the variables. Nevertheless, even in cases for which it is inappropriate as a representation, linear mathematics provides us with useful tools for analysis and a guide in our search for analogous nonlinear techniques.

The only widely used nonlinear approach to biochemical systems is that provided by the Michaelis-Menten Formalism. ${ }^{2}$ This approach too has its attractions. It has been shown to provide a good approximation to the rate law for many specific enzyme-catalyzed reactions in vitro, and it presents well-recognized procedures for estimating the parameter values in simple cases. One of the principal disadvantages lies in the fact that it is not a general formalism-general in the sense that there is an explicit mathematical structure within which all the special cases are contained. Each system

\footnotetext{
2By the Michaelis-Menten Formalism we do not mean just the original MichaelisMenten assumptions, derivation and specific rate law [18] but the broader spectrum of subsequent developments in enzyme kinetics that nonetheless share key assumptions and empirical methodology (e.g., [19, 15, 3]). For example, the assumption that there are no interactions between the various enzyme forms in a mechanism or between forms from other enzymes, yields steady-state equations that are linear in the concentrations of the enzyme forms [3]. The solution of these equations produces rate laws that are linearly related to the concentration of total enzyme $[48,22]$.
} 
must be developed on an ad hoc basis. Such systems are difficult to analyze symbolically and to compare with alternatives; furthermore, computer implementation is unsystematic and conventional general-purpose algorithms often are expensive to run. (For further discussion see [41].)

Fruitful development of a general nonlinear formalism-general in the sense given above-that would retain many of the advantages of these existing alternatives began with the hint that rational functions, which provide a good representation for many rate laws in vitro, often can be approximated over a wide range by a straight line in a log-log plot [2]. This property suggested the Power-Law Formalism [22, 23], analogous to the Linear Formalism, based on Taylor's Theorem ${ }^{3}$ but applied in a logarithmic space [27]. Thus, the rate law for a reaction or process is approximated by the first two terms of its Taylor series in logarithmic space.

$$
\begin{aligned}
\log v_{i}\left(X_{1}, \ldots, X_{n}\right)= & \log v_{i}\left(X_{1 O}, \ldots, X_{n O}\right) \\
& +\sum_{j=1}^{n} \frac{\partial\left[\log v_{i}\left(X_{1 O}, \ldots, X_{n O}\right)\right]}{\partial\left[\log X_{j}\right]}\left(\log X_{j}-\log X_{j O}\right) \\
& +\cdots
\end{aligned}
$$

where $v_{i}\left(X_{1}, \ldots, X_{n}\right)$ is the rate of the reaction or process in question, and the variables $X_{j}$ are concentrations of enzymes, reactants, or modifiers, or any other variables that affect the process. The additional $O$ subscript signifies evaluation about a given operating point, and, in the case of derivatives, evaluation at the operating point after performing the appropriate partial differentiation. By regrouping terms, Equation (1) can be rewritten as

$$
\log v_{i}\left(X_{1}, \ldots, X_{n}\right)=\log \alpha_{i}+g_{i 1} \log X_{1}+\cdots+g_{i n} \log X_{n}
$$

and when it is transformed back into Cartesian coordinates, it can be expressed as a product of power-law functions [23].

$$
v_{i}\left(X_{1}, \ldots, X_{n}\right)=\alpha_{i} \prod_{j=1}^{n} X_{j}^{g_{i}}
$$

\footnotetext{
${ }^{3}$ Recall that Taylor's Theorem is among the most general and powerful tools for applied mathematical analysis because it has few restrictions and these are generally satisfied for any real system of interest. It states that any continuously differentiable function can be represented by a finite power series with any desired degree of accuracy, which is specified by the remainder term. (For example, see [38] for details.)
} 
where

$$
g_{i, j}=\partial\left(\log v_{i O}\right) / \partial\left(\log X_{j}\right)=\left(\partial v_{i O} / \partial X_{j}\right)\left(X_{j O} / v_{i O}\right)
$$

and

$$
\boldsymbol{\alpha}_{i}=v_{i O} \prod_{i=1}^{n} X_{i j}^{-g_{i}}
$$

In chemical and biochemical kinetics the $g_{i j}$ exponents and the $\alpha_{i}$ coefficient in Equation (2) traditionally have been called kinetic orders and rate constant. Equation (2) is important because it gives explicitly the structural relationship of flux to the variables and to the fundamental parameters of a process near a given operating point. It should be emphasized that this formalism is valid for any function and for all types of variables, provided that the logarithmic derivatives exist and the excursions of the variables about their nominal values are small.

BST is built explicitly upon this underlying Power-Law Formalism. However, it should be emphasized that this Formalism is more general than BST. As we shall see in Section 3.1, BST is only one of many realizations (see also [41]).

The Power-Law Formalism has not been identified explicitly in MCT. Nevertheless, it is clear that MCT is based upon an important special case. Ad hoc definitions of elasticity [12] and effector strength [10] as relative change in reaction rate with respect to relative change in metabolite concentration (pools linking reactions) in fact are special cases of kinetic order $[23,25]$. Though no definition corresponding to that of rate constant $[23,25]$ has been proposed in MCT, a special case of the general Power-Law Formalism can be scen to apply. If the ratc law is proportional to enzyme concentration (or molecular activity) and if the rate laws are independent [13], as may be true for many but not all enzymes (see comments above and $[32,41,44])$, then the enzyme concentration has a kinetic order of unity. Furthermore, if the enzyme level is considered a parameter and not a variable, then its symbol can be absorbed into the rate constant $\alpha_{i}$ (see [23, 27]). The parameter $\alpha_{i}$ then will be directly proportional to enzyme concentration. This is the case upon which MCT is built $[12,10,13]$.

Although the molecular descriptions in MCT have not been defined explicitly, the explicit structural relationship in the Power-Law Formalism can be written in the current notation of MCT as

$$
v_{i}\left(X_{1}, \ldots, X_{n}\right)=E_{i} \prod_{j=1}^{n} X_{j}^{\epsilon_{X_{j}}}
$$


where the variables $X_{j}$ refer to metabolite concentrations and $E_{i}$ is proportional to enzyme (or molecular) activity. ${ }^{4}$ Although the rate constant parameter previously has not been defined explicitly in MCT, the above convention will yield results identical to those previously reported in MCT, where $E_{i}$ is given the more restricted definition of enzyme (or molecular) activity $[12,13]$.

Equations (2) and (5) have the same form; they differ only with regard to their underlying assumptions and differences in interpretation. Thus, the Power-Law Formalism underlies both BST and MCT, and the local descriptions in MCT comprise a special case of those in BST. If we generalize the molecular descriptions in MCT, it is clear that they can be equated with those in BST. Then, at least with regard to molecular descriptions, the differences in terminology are merely a matter of one-for-one translation.

\subsection{IMPLICATIONS FOR PARTICULAR APPLICATIONS}

Although methods for measuring specific parameter values are not a part of the general thcory, it is important in particular applications of the theory to know how such measurements can be accomplished. A familiar example will make the meaning and measurement of the two types of fundamental parameters clear [25]. The traditional Michaelis-Menten rate law is given by

$$
v=V_{m} X /\left(K_{m}+X\right)
$$

where $v$ is the reaction velocity, $X$ is substrate concentration, $V_{m}$ is the maximal velocity and $K_{m}$ is the Michaelis constant. According to Equations (3) and (4)

$$
g=\begin{aligned}
& \partial v_{O} X_{O} \\
& \partial X v_{O}^{v_{O}}
\end{aligned}=\frac{K_{m} V_{m}}{\left(K_{m}+X_{O}\right)^{2}}{ }^{K_{m}+X_{O}} V_{m}=\begin{gathered}
K_{m} \\
K_{m}+X_{O}
\end{gathered}
$$

and

$$
\alpha=\frac{V_{m} X_{O}}{K_{m}+X_{O}} X_{O}^{-K_{m} /\left(K_{m}+X_{O}\right)}
$$

where $X_{O}$ is the nominal operating value of $X$ and subscripts for $g$ and $\alpha$

\footnotetext{
${ }^{4}$ One easily can verify that this explicit structural relationship underlies the local descriptions in MCT by calculating the derivatives that define the elasticity parameters in MCT and showing that the results are identical to the exponents in Equation (5), i.e.,
}

$$
\left(\partial v_{i} / \partial X_{i}\right)\left(X_{j} / v_{i}\right)=\epsilon_{X_{i}}^{\prime}, \quad\left(\partial v_{i} / \partial E_{i}\right)\left(E_{t} / v_{i}\right)=1, \quad \text { and }\left(\partial v_{i} / \partial E_{j}\right)\left(E_{j} / v_{i}\right)=0 .
$$


have been omitted for simplicity. At substrate concentrations much less than $K_{m}\left(X_{O} \ll K_{m}\right): g \doteq 1$ and $\alpha \doteq V_{m} / K_{m}$, which is a linear function of $X$ with slope $V_{m} / K_{m}$. At substrate concentrations approximately equal to $K_{m}$ $\left(X_{O}=K_{m}\right): g \doteq \frac{1}{2}$ and $\alpha \doteq V_{m} / 2 K_{m}^{\frac{1}{2}}$, which is a square-root function. At substrate concentrations much greater than $K_{m}\left(X_{O} \gg K_{m}\right): g \doteq 0$ and $\alpha \doteq V_{m}$, which is a constant function equal to $V_{m}$.

Thus, the values for the molecular parameters $g$ and $\alpha$ can be obtained by direct mathematical calculation when the relevant rate laws and operating values are known [25]. They also can be obtained graphically by plotting in $\log$ - $\log$ coordinates the reaction velocity as a function of substrate concentration, following small variations about the appropriate in vivo conditions. The slope gives the value of $g$ directly and the value of $\alpha$ easily can be determined from the intercept on the $\log v$-axis [23, 25, 27]. Experimental evidence from a number of systems indicates that the range of variation over which Equation (2) remains valid is remarkably wide [25, 29 Ch. 51314,31 , 33], which makes the experimental measurement of the parameters in Equation (2) practical. (This point is examined in more detail elscwherc [41].)

The first method of measuring kinetic orders has been adopted primarily by Heinrich and colleagues (e.g., see [10,21]), while the second method has been adopted primarily by Kacser and coworkers (e.g., see [12, 7]) in their applications of MCT to particular systems. In any case, one of the principle advantages of the Power-Law Formalism recognized very early was that one need not be engulfed by detailed kinetic analysis; one could make a few measurements after small deviations under in vivo conditions and determine the key parameters in the formalism [24, 25, 27].

\subsection{IMPLICATIONS FOR THE GENERAL THEORY}

Note that in the derivation of Equations (1-4):

1. No assumptions are made with regard to the detailed character of the rate law. The only assumption made is that the rate law be a logarithmically differentiable function, which is true for virtually any system of interest. (We will have more to say about this elsewhere.)

2. Equation (2) can be written for any set of operating values; of course the values of the parameters will change according to the operating conditions (e.g., see $[23,25]$ ).

3. For small deviations about a given set of operating values, Fquation (2) is guaranteed by Taylor's Theorem to be an accurate representation of the true rate law and the parameters are constants.

4. The representation in Equation (2) contains all the information concerning the operating point (e.g., a steady state of the system) and the first derivatives of the rate law with respect to the relevant variables at this operating point. For a rate law or process involving $n$ variables, there are 
$n+1$ fundamental parameters that uniquely characterize the process under these conditions. If one is concerned only with the value and first derivatives of a rate law (e.g., as is the case in MCT), then Equation (2) is an exact representation of the actual rate law. That is, if one is concerned only with behavior in response to infinitesimal changes, then one can work with either the actual rate laws or their power-law representations throughout and the conclusions will be identical.

Thus, it can be seen that the Power-Law Formalism provides a very general theoretical foundation for a biochemical systems theory, and that it underlies both BST and MCT.

In BST the Power-Law Formalism has provided a solid theoretical foundation and has played an explicit role throughout the development of the theory [23, 31]. All molecular parameters were rigorously defined in terms of this formalism, as were all the global factors that will be discussed fully in the following paper [34]. The formalism, and not an ad hoc choice, dictated the appropriate definition of parameters. In MCT, the Power-Law Formalism was implicit in the assumptions of a steady state, logarithmically differentiable rate laws and of infinitesimal variation $[12,10]$. That is, MCT has been concerned only with the value and first derivatives of the rate laws and thus has used only the information explicitly present in the kinetic orders and rate constants as defined above. The local parameter values and global factors in MCT were defined in terms of relative changes to avoid the units of measurement [12,10], and, fortuitously, these parameters and factors were identical to those defined earlier in BST [23, 25, 26].

One can conclude that, with regard to the molecular description of the elements of biochemical systems, MCT, when suitably generalized to include the rate constant parameter and variables other than metabolite concentrations, is identical to BST and that MCT is thus a rediscovery of BST. Nevertheless, there are differences in interpretation (even in the molecular descriptions) that stem from the fact that the underlying structure is explicit in BST but implicit in MCT. These differences are minor when compared with the more fundamental differences between BST and MCT that pertain to the way molecular descriptions are combined to yield global descriptions of intact systems and to their treatment or non-treatment of local dynamics. These issues will be taken up next.

\section{CHARACTERIZATION OF INTACT SYSTEMS}

\subsection{AGGRLGATION OF FLUX}

In BST, rate laws for those processes tending to increase a given substance are first summed to produce a net or aggregate rate law. Similarly, rate laws for those tending to decrease a given substance are summed to 
produce a separate net or aggregate rate law. Then the Power-Law Formalism is used to represent explicitly each of these net rate laws, and equations can be written to describe the intact system. There is one equation for each of the $n$ dependent variables, which may be thought of as variables "internal" to the system. If there are $m$ independent variables, which may be considered external variables that are determined by factors outside the system of interest (e.g., by the experimentalist, the environment, or other systems within the same organism), then

$$
d X_{i} / d t=\alpha_{i} \prod_{i=1}^{n+m} X_{j}^{g_{i}}-\beta_{i} \prod_{j=1}^{n+m} X_{j}^{h_{1+}} \quad i=1, \ldots, n .
$$

Two new symbols have been introduced: $\beta_{i}$ and $h_{i j}$. The symbol $\beta_{i}$ is the rate constant for the net decrease of $X_{i}$ and $h_{i j}$ is the kinetic order for the net decrease of $X_{i}$ with respect to variation in $X_{j}$. Thus, the symbols $\beta$ and $\alpha$ both represent rate constants, and the symbols $h$ and $g$ both represent kinetic orders; the distinction is that $\beta$ and $h$ are reserved for the processes that tend to decrease $X_{i}$ while $\alpha$ and $g$ are reserved for the processes that tend to increase $X_{i}$. (For more detailed discussion of dependent and independent variables and the algorithm for writing the system equations see [27, $29 \mathrm{Ch}$. 9] and [40]. This convention is based on a unified numbering scheme - provided by the variables - in which each parameter is identified immediately and unambiguously and can be related directly to the underlying structure or topology of the system.

By contrast, the underlying Power-Law Formalism has not been recognized in MCT. Therefore, we must make this aspect of MCT explicit in order to reveal the fundamental differences in the ways that molecular descriptions are combined to yield systemic descriptions. The net flux for individual enzyme-catalyzed reactions is the unit of aggregation, and independent rate laws are assumed for each with no further aggregation. Infinitesimal relative variations then are assumed. This allows the functional representation of the rate laws to be replaced by their power-law representation, which under the assumed conditions is an exact representation of the actual rate laws. There is one equation for each of the $n$ dependent concentration variables in the system, and the equations corresponding to Equation (8) can be written with a generalization of the current notation in MCT as

$$
d X_{i} / d t=\sum_{r} E_{r} \prod_{j=1}^{n+m} X_{j}^{\epsilon^{r} X_{j}}-\sum_{s} E_{s} \prod_{j=1}^{n+m} X_{j}^{\epsilon^{s} X_{j}} \quad i=1, \ldots, n
$$

where the summation in each case is over the relevant reactions. It should be noted that the numbering scheme in MCT is a dual system-concentration variables (indices $i$ and $j$ ) are numbered independently of the reactions and 
enzymes (indices $r$ and $s$ ). In general, an extra connectivity matrix (e.g., see [46]) is needed to relate these two independent sets of numbers to each other and thus to the underlying structure of the system. The different numbering systems must be kept clearly in mind when comparing results obtained with BST and MCT, respectively.

It is explicitly assumed in MCT that reaction rate is proportional to enzyme concentration or molecular activity, which are considered parameters of the system $[12,10,13]$. The rate constant parameters $E_{r}$ and $E_{s}$ are proportional to enzyme concentration or molecular activity as noted for Equation (5). Thus, in the current version of MCT one cannot represent enzyme-proenzyme cascades, certain hormonal systems, or the regulation of gene expression, for the enzyme levels in these types of systems are not fixed parameters or independent variables. Rather they are dependent concentration variables whose values are determined by the values of the independent variables and parameters of the system $[25,27]$. If one generalizes the current interpretations of MCT, as we did in Section 2.1, then cascade mechanisms can be represented by Equation (9). The analogy to the corresponding treatment in BST is straightforward. However, care must be taken with the dual numbering system in MCT, for enzyme concentrations and reactions in the current version of MCT are enumcrated separately from the variable (metabolite) concentrations.

It must be emphasized that these two representations-Equation (8) for BST and Equation (9) for generalized MCT - are equally valid for infinitesimal variations about a steady state for the system. In fact, they may be viewed as two different forms of aggregation on a spectrum from no aggregation, representing elemental chemical kinetic steps in the individual mechanisms, to aggregation of an entire system, all of which are equally valid for infinitesimal variations about a steady state [41]. Nevertheless, as we shall see, there are distinct advantages to aggregation in the form of Equation (8), and these make BST a more general and systematic theory.

\subsection{AGGREGATION OF CONCENTRATION}

Aggregate variables in biochemical systems, and indeed any algebraic constraints among the variables of such systems, are treated in BST no differently than the power-law description of the component processes themselves [23, 30]. In general,

$$
X_{t}=f\left(X_{1}, \ldots, X_{j}, \ldots\right)
$$

and the expression is represented in the Power-Law Formalism as

$$
X_{t}=\gamma_{t} \prod_{j} X_{j}^{f_{t j}}
$$


where

$$
f_{t j}=\left(\partial X_{t o} / \partial X_{j}\right)\left(X_{j o} / X_{t o}\right)
$$

and

$$
\gamma_{t}=X_{t o} \prod_{j} X_{j o}^{-f_{t j}}
$$

A common example is

$$
X_{t}=\sum_{j} X_{j}
$$

where the sum over the relevant dependent variables is an aggregate variable; in general the function need not be a simple sum. The aggregate variable $X_{t}$ may represent a fundamental variable such as the total adenylate concentration, the total cofactor concentration, or the sum of free and bound forms of an enzyme. It may be considered either an independent variable of the system or a dependent variable; in either case, each such constraint reduces by one the number of differential equations for the system (Equation 8). Aggregate variables need not be fundamental variables of the system or constraints upon the system. They simply may be sums of fundamental variables that happen to be convenient quantities for measurement.

For the specific case in Equation (11), note that the exponent $f_{t j}$ is simply the fraction of the aggregate represented by $X_{j O}$ in the steady state, i.e., $f_{t, j}=X_{j o} / X_{t o} .^{5}$

If, for example, $X$, is an independent variable representing the sum of two forms of a cofactor whose total amount is conserved, then either of the two dependent variables in the aggregate can be expressed in terms of the remaining variable by rearranging Equation (10). This rearranged algebraic equation then can be substituted into the remaining differential equations, thereby eliminating one of the dependent variables from the description of the system (see [30] for details). The resulting system of differential equations then can be expressed in the same form as Equation (8).

Aggregate variables have only recently been treated within MCT. Westerhoff and Chen [46] have dealt with the specific problem of a constant adenylate pool by defining an elasticity for the ratio of ATP to ADP concentrations. Fell and Sauro [6] have treated this and other specific cases by an alternative approach, which is equivalent to the general treatment in BST first published in 1979 [30]. We shall not deal explicitly with aggregate

\footnotetext{
${ }^{5}$ The power-law representation of aggregate variables is formally analogous to that of conventional rate laws, although general algebraic constraints are being represented. By convention, the parameters $\gamma$ and $f$ are analogous to $\alpha$ (or $\beta$ ) and $g$ (or $h$ ).
} 
concentration variables in this paper because, as indicated above, systems with these types of variables can be reformulated and then treated in exactly the same fashion as systems without such variables.

\subsection{PROPERTIES OF STEADY-STATE SOLUTIONS}

There is no way to obtain an explicit steady-state solution for the general balance or conservation equations (e.g., Equation (1) in [23] and Equation (A1) in [12]) that characterize a system when these involve arbitrary nonlinear rate laws. Even specific numerical solutions are not always feasible for such equations. These pronounced difficulties for any general theory are surmounted with the Power-Law Formalism, provided one chooses the appropriate form of aggregation.

In steady state, the time derivatives are equal to zero, and in BST Equation (8) can be written in conventional matrix notation as

$$
[\mathrm{A}] \mathrm{y}]=\mathbf{b}]
$$

where

$$
\begin{aligned}
y_{i} & =\log X_{i} \\
b_{i} & =\log \left(\beta_{i} / \alpha_{i}\right) \\
a_{i j} & =g_{i j}-h_{i j} .
\end{aligned}
$$

In MCT there is no simple representation equivalent to Equation (12); the logarithmic transformation of Equation (9) does not produce a linear system because of the form of aggregation used.

Existence Theorem. A biochemical system described by Equations (8) and (12) has a positive steady-state solution provided

$$
\operatorname{rank}[\mathbf{A}]=n \text {. }
$$

If the system is autonomous (i.e., it has no external variables), then Equation (13) can be expressed as

$$
|\mathbf{A}| \neq 0
$$

where $|\mathbf{A}|$ is the determinant of the matrix [A]. Thus, an $n \times n$ determinant of the differences between the corresponding kinetic orders for net increase and net decrease-and only these molecular parameters-determines whether or not a positive steady-state solution for the system will exist [23]. Conversely, the existence of such a steady state implies that the kinetic orders of the system cannot have any values, but are constrained by Equation (14). This is a general theorem that holds regardless of the underlying rate laws and operating conditions that may exist. 
In MCT there is no corresponding existence theorem that can be obtained explicitly in a general symbolic form for the steady-state solutions of Equation (9).

Explicit Solutions. When the existence theorem in BST is satisfied, Equation (12) can be solved to yield the internal (or dependent) variables as an explicit function of the parameter values and the external (or independent) variables of the system [25]:

$$
\left.\left.\mathbf{y}]_{\text {in }}=[\mathbf{L}] \mathbf{y}\right]_{\mathrm{ex}}+[\mathbf{M}] \mathbf{b}\right]
$$

where $y]_{\text {in }}$ and $\left.y\right|_{e x}$ are vectors whose elements are the logarithms of the internal and external variables, $[\mathbf{L}]$ and $[\mathbf{M}]$ are matrices determined by inversion of the underdetermined system in Equation (12) and composed of elements that are functions only of the kinetic orders, and b] is a vector whose elements are functions only of the rate constants (c.f. Equation 12). This general symbolic solution exhibits the structure of the relationships between molecular parameters and variables in an explicit form, and it provides a direct method for further characterization of systemic behavior in steady state. Furthermore, this approach applies without modification to arbitrary topologics, including branched as well as unbranched systems [27].

The behavior of the dependent variables was separated deliberately into these two components and represented by distinct symbols to emphasize the influence of the independent variables and the parameters of the system. The independent variables may be thought of as those that are determined by factors outside the system of interest. The parameters, which characterize the relatively fixed aspect of the system itself, may be thought of as physically and genetically determined. To use an analogy, the music emanating from a stereo system is a function of the externally supplied (by tape or record) stimuli, which are variables, and of the system parameters, which are relatively fixed and determined by the electro-mechanical components of the system. The separation of these two types of influences is important to a clear understanding of system behavior.

From Equation (8) and the solution in Equation (15) the net fluxes follow trivially:

$$
\log \mathbf{V}]=\log \alpha]+[\mathbf{G}] \mathbf{y}]
$$

where $V_{i}$ is the net rate of synthesis of $X_{i}, \alpha_{i}$ is the corresponding rate constant, and the matrix $[\mathbf{G}]$ is composed of the $g_{i}$ kinetic orders. From the general symbolic solution in Equations (15) and (16) one can calculate directly systemic properties and exhibit their relation to the parameters of the underlying molecular mechanisms, as was first shown by Savageau [25]. These same systemic or global properties also can be obtained graphically from appropriate experimental data plotted in $\log \log$ coordinates [25, 29]. 
In MCT steady-state solutions cannot be obtained explicitly in a general symbolic form analogous to Equations (15) and (16), but only numerically in specific cases, and this may not always be feasible. Furthermore, the original development of MCT applied only to simple unbranched systems [12, 10]; auxiliary conditions that have been developed subsequently [11] were required to deal with branched systems.

\section{DISCUSSION}

MCT has developed as a collection of ad hoc definitions, assumptions and implicit methods rather than as a general, explicitly structured theory from first principles. Our first task has been to show that MCT is in fact based upon the Power-Law Formalism, the same formalism that underlies BST. At this level one can discern easily the fundamental similarities and differences between BST and MCT.

The molecular descriptions in MCT are identical to those first described in BST. The only differences pertain to interpretation. In BST all the parameters are defined in terms of Taylor's Theorem in logarithmic coordinates and are operationally meaningful. In MCT the molecular parameters were defined in an ad hoc fashion to eliminate consideration of measurement units. Elasticities (or effector strengths) proved to be identical to the kinetic orders in BST. In contrast, the rate constant parameter has not been defined in MCT. Reaction rates are assumed in MCT to be linearly related to enzyme concentrations and molecular activities, and the consequences of variation in these parameters, which are then proportional to the rate constant, have been examined $[12,10]$. While these are useful simplifying assumptions that can be made in either BST or MCT whenever they are appropriate, they are not generally valid (see Section 2) and thus cannot form the basis for a general theory.

The systemic descriptions in MCT and BST differ with regard to the level of aggregation assumed. In BST, aggregation is at the level of net rates of increase and net rates of decrease of each system constituent. This level of aggregation was selected in the original development [23] because this choice was found to produce a theory with the most fruitful and convenient mathematical structure. This manifestation reveals, among other things, the fundamental importance of differences between the kinetic orders for net increase and for net decrease; these differences appear repeatedly in the general theory. The existence theorem for a steady-state solution (Equation 13) is expressed naturally in these differences. Explicit steady-state solutions in a general symbolic form can be expressed in these differences (Equations 15 and 16). (Other examples will be given elsewhere [34].) The general symbolic solution contains all information necessary to characterize directly the relationships between systemic and molecular properties, which has made it possible to draw general conclusions that are independent of particular parameter values for entire classes of systems (e.g., see [28]). 
In MCT, aggregation at the level of rate laws for individual enzyme-catalyzed reactions has been assumed. In the corresponding manifestation of the Power-Law Formalism there is neither a simple existence theorem for a steady-state solution analogous to Equation (12) nor an explicit steady-state solution in a general symbolic form analogous to Equations (15) and (16). Because MCT provides no direct method for calculating the general relationships between systemic and molecular properties, MCT has given rise to another, more indirect, approach based on the summation and connectivity relationships first described by Kacser and Burns [12] and Heinrich and Rapoport [10]. This approach will be the subject of the following paper [34].

Recent results [41] have shown that the aggregate descriptions in BST are valid over a wider range of variation in the concentration variables than are the corresponding descriptions in MCT. These results suggest that when finite variations are employed under in vivo conditions to estimate molecular or systemic properties, the values determined will be more accurate in BST.

The above paragraphs summarize the results of our comparison of the fundamental structures of BST and MCT. From these results a number of conclusions follow. At this fundamental level, and thus with regard to actual results of applications, BST and MCT are identical over the domain of MCT. In this fundamental sense MCT represents a rediscovery of the earlier BST. Moreover, it is the rediscovery of a special case of BST, for the content and range of validity of BST are greater than those of MCT. Thus, BST is seen to be the richer theory in terms of content and structure available for further theoretical development (see also [34, 41]).

If this were the only issue, there would be little interest in attempting to perpetuate MCT as a separate entity. However, the most intuitively attractive aspects of MCT - the summation and connectivity relationships-have yet to be addressed. These will be the subject of the following paper.

This work was supported in part by U.S. Public Health Service Grant $G M-30054$ from the National Institutes of Health. The material presented in this paper was part of a larger manuscript circulated among Drs. R. Heinrich, H. Kacser, J. Porteous, S. Rapoport and H. Westerhoff in March 1985. We thank them for their useful comments.

\section{REFERENCES}

1 L. von Bertalanffy, General Systems Theory, George Braziller, New York, 1968.

2 H. W. Bode, Network Analysis and Feedback Amplifier Design, Van Nostrand, Princeton. New Jersey, 1945.

3 W. W. Cleland, Enzyme kinetics, Ann. Rev. Biochem. 36:77-112 (1967).

4 J. B. Cruz, ed., System Sensitivity Analysis, Dowden, Hutchinson and Ross, Stroudsburg, Pennsylvania, 1973

5 R. H. Davis, Channeling in Neurospora metabolism, in Organizational Biosynthesis (H. J. Vogel, J. O. Lampen, and V. Bryson, Eds.), Academic Press, New York, 1967, pp. 303-322. 


\section{MICHAEL A. SAVAGEAU, EBERHARD O. VOIT, AND DOUGLAS H. IRVINE}

6 D. A. Fell and H. M. Sauro, Metabolic control and its analysis, Eur. J. Biochem. 148:555-561 (1985).

7 H. J. Flint, R. W. Tateson, I. B. Barthelmess, D. J. Porteous, W. D. Donachie, and H. Kacser, Control of the flux in the arginine pathway of Neurospora crassa, Biochem. J. 200:231-246 (1981).

8 C. Frieden, Slow transitions and hysteretic behavior in enzymes, Annu. Rev. Biochem. 48:471-489 (1979).

9 C. N. Hankins and S. E. Mills, A dimer of a single polypeptide chain catalyzes the terminal four reactions of the L-tryptophan pathway in Euglena gracilis, J. Biol. Chem. 252:235-239 (1977).

10 R. Heinrich and T. A. Rapoport, A linear steady-state treatment of enzymatic chains: Gencral properties, control and effector strength, Eur. J. Biochem. 42:89-95 (1974).

11 R. Heinrich and T. A. Rapoport, Mathematical analysis of multienzyme systems 2. Steady-state and transient control, Biosystems 7:130-136 (1975).

12 H. Kacser and J. A. Burns, The control of flux, Symp. Soc. Exp. Biol. 27:65-104 (1973).

13 H. Kacser and J. W. Porteous, Control of metabolism: What do we have to measure?, Trends Biochem. Sci. 12:5-14 (1987).

14 J. F. Kane, Regulation of a common amidotransferase subunit. J. Bacteriol. $132: 419-425$ (1977).

15 D. E. Koshland, G. Nemethy, and D. Filmer, Comparison of experimental binding data and theoretical models in proteins containing subunits, Biochemistry 5:365-385 (1966).

16 B. I. Kurganov, The theoretical analysis of kinetic behavior of "hysteretic" allosteric enzymes: IV. Kinetics of dissociation-association processes of allosteric enzymes, $J$. Theoret. Biol. 68:521-543 (1977).

17 L. J. Meyer and M. A. Becker, Human erythrocyte phosphoribosylpyrophosphate synthetase, J. Biol. Chem. 252:3919-3925 (1977).

18 L. Michaclis and M. L. Menten, Die Kinetik der Invertinwirkung, Biochem. 7. 49:333-369 (1913).

19 J. Monod, J. Wyman, and J.-P. Changeux, On the nature of allosteric transitions: A plausible model, J. Mol. Biol. 12:88-118 (1965).

$20 \mathrm{~J}$-C. Patte, P. 'Truffa-Bach, and $\dot{G}$. N. Cohen, I he threonine-sensitive homoserine dehydrogenase and aspartokinase activities of Escherichia coli. I. Evidence that the two activities are carried by a single protein, Biochim. Biophys. Acta 128:426-439 (1966).

21 T. A. Rapoport, R. Heinrich, and S. M. Rapoport, The regulatory principles of glycolysis in erythrocytes in vivo and in vitro, Biochem. J. 154:449-469 (1976).

22 M. A. Savageau, Biochemical systems analysis. I. Some mathematical properties of the rate law for the component enzymatic reactions, J. Theoret. Biol. 25:365-369 (1969).

23 M. A. Savageau, Biochemical systems analysis. II. The steady-state solutions for an n-pool system using a power-law approximation, J. Theoret. Biol. 25:370-379 (1969).

24 M. A. Savageau, Biochemical systems analysis. III. Dynamic solutions using a powerlaw approximation, J. Theoret. Biol. 26:215-226 (1970).

25 M. A. Savageau, Concepts relating the behavior of biochemical systems to their underlying molecular properties, Arch. Biochem. Biophys. 145:612-621 (1971).

26 M. A. Savageau, Parameter sensitivity as a criterion for evaluating and comparing the performance of biochemical systems, Nature 229:542-544 (1971).

27 M. A. Savageau, The behavior of intact biochemical control systems, Curr. Top. Cell. Reg. 6:63-130 (1972). 
28 M. A. Savageau, Optimal design of feedback control by inhibition: Steady-state considerations. J. Mol. Evol. 4:139-156 (1974).

29 M. A. Savageau. Biochemical Sistems Analysis: A Study of Function and Design in Molecular Biologl. Addison-Wesley, Reading, Massachusetts, 1976.

30 M. A. Savageau, Growth of complex systems can be related to the properties of their underlying determinants. Proc. Natl. Acad. Sci. USA 76:5413-5417 (1979).

31 M. A. Savageau. Mathematics of organizationally complex systems, Biomed. Biochim. Acra 44:839-844 (1985).

32 M. A. Savageau. Control of metabolism: Where is the theory?, Trends Biochem. Sci 12:219-220 (1987).

33 M. A. Savageau and E. O. Voit. Power-law approach to modeling biological systems I. Theory, J. Ferment. Technol. 60:221-228 (1982).

34 M. A. Savageau, E. O. Voil, and D. H. Irvine, Biochemical systems theory and metabolic control theory: 2 . The role of summation and connectivity relationships, Math. Biosci., 86:147-169 (1987).

35 S. C. Singer and F. W. Holmes. Human glutamine phosphoribosylpyrophosphate amidotransferase, J. Biol. Chem. 252:7959-7963 (1977).

36 P. A. Singer, M. Levinthal, and L. S. Williams, Synthesis of the isoleucyl- and valyl-tRNA synthetases and the isoleucine-valine biosynthetic enzymes in a threonine deaminase regulatory mutant of Escherichia coli K-12, J. Mol. Biol. 175:39-55 (1984).

37 D. K. Srivastava and S. A. Bernhard, Metabolite transfer via enzyme-enzyme complexes. Science 234:1081-1086 (1986).

38 G. B. Thomas, Jr. and R. L. Finney, Calculus and Analytic Geometry, 5th ed Addison-Wesley, Reading, Massachusetts, 1982.

39 E. O. Voit. Control in perspective, Trends Biochem. Sci. 12:221 (1987).

40 E. O. Voit and M. A. Savageau, Power-law approach to modeling biological systems III. Methods of analysis, J. Ferment. Technol. 60:233-241 (1982).

41 E. O. Voit and M. A. Savageau, Accuracy of alternative representations for integrated biochemical systems, Biochemistry, to appear.

42 R. J. A. Wanders, A. K. Groen, C. W. T. van Roermund, and J. M. Tager, Factors determining the relative contribution of the adenine-nucleotide translocator and the ADP-regenerating system to the control of oxidative phosphorylation in isolated rat-liver mitochondria, Eur. J. Biochem. 142:417-424 (1984).

43 G. R. Welch, ed., Organized Multienzyme Systems: Catalytic Properties, Academic Press, New York, 1985.

44 G. R. Welch and T. Keleti, Is cell metabolism controlled by a 'molecular democracy' or by a 'supramolecular socialism'?, Trends Biochem. Sci. 12:216-217 (1987).

45 G. R. Welch, T. Keleti, and B. Vertessy. The control of cell metabolism for homogeneous vs. heterogeneous enzyme systems, $J$. Theoret. Biol, to appear.

46 H. V. Westerhoff and Y.-D. Chen, How do enzyme activities control metabolite concentrations?, Eur. J. Biochem. 142:425-430 (1984).

47 J. M. Wiame, The regulation of arginine metabolism in Saccharomyces cerevisiae: Exclusion mechanisms, Curr. Top. Cell. Reg. 4:1-38 (1971).

48 J. T.-F. Wong and C. S. Hanes, Kinetic formulations for enzymatic reactions involving two substrates. Can. J. Biochem. Physiol. 40:763-804 (1962). 